\title{
Serum levels of high sensitivity C-Reactive protein and its association with lipidemic status in Bangladeshi healthy adults
}

\author{
Rehnuma B ${ }^{1}$, Hassan Z ${ }^{2}$, Ibrahim $\mathrm{M}^{3}$, Ali L ${ }^{4}$ \\ ${ }^{I}$ Department of Lab Medicine, Apollo Hospitals, Dhaka, Bangladesh. \\ ${ }^{2}$ Department of Physiology and Molecular Biology, BIRDEM, Dhaka, Bangladesh. \\ ${ }^{3}$ Department of Lab Medicine, Apollo Hospitals, Dhaka, Bangladesh. \\ ${ }^{4}$ Department of Biochemistry and Cell Biology, BIRDEM, Bangladesh.
}

\section{Keywords:}

High sensitive C-reactive protein;

Metabolic disorders;

Lipids.

\begin{abstract}
Background: Metabolic diseases are affecting human health all over the world. These chronic disease states are associated with high sensitive CRP. The present study aims to measure hsCRP and lipid levels in a group of healthy adults to work out the normal hsCRP levels and explore its relationship to lipidemic and anthropometric variables.
\end{abstract}

Materials and Methods: A total of 149 adults, age 30-60 yrs, healthy subjects were recruited in this study. Vital statistics were taken along with measurement of blood glucose, triglyceride, total cholesterol, high density lipoprotein, low density lipoprotein and hsCRP levels. Data were analyzed using statistical Package for Social Program (SPSS) for Windows version 10.

Results: Mean $( \pm \mathrm{SD})$ hsCRP was $2.46 \pm 1.89(\mathrm{mg} / \mathrm{l})$ with a cut off value of $3 \mathrm{mg} / \mathrm{l}$. Of the total 149 subjects $102(66.4 \%)$ and 47 (31.5\%) subjects had hsCRP level below and above the cutoff respectively. Triglyceride was significantly higher $(\mathrm{p}=0.033)$ and HDL was lower in subjects with high hsCRP. Total cholesterol and LDL was almost similar in both groups. Binary logistic regression showed significant negative association between hsCRP and HDLc $(\mathrm{p}=0.029)$ and but the model excluding HDL-c showed significant positive association $(\mathrm{p}=0.03)$ with triglyceride.

Conclusion: About one-third of healthy Bangladeshi adults may have chronic subclinical inflammation and thus may have a risk of cardiovascular disorders. More than one-third healthy Bangladeshi adults have one or more type of dyslipidemia and the subclinical chronic inflammation in healthy Bangladeshi adults has a positive association with their serum triglyceride and negative association with their HDLcholesterol levels.

\section{INTRODUCTION}

C-reactive protein (CRP) is an inflammatory marker whose expression is markedly up regulated during inflammation. ${ }^{1}$

\section{Correspondence:}

Dr Behterin Rehnuma, M.Phil

Registrar, Clinical Biochemistry

Lab Medicine Department, Apollo Hospitals, Dhaka, Bangladesh

Email:planet_behterin@yahoo.com
It is the acute phase protein synthesized in the liver and regulated to a large extend by pro-inflammatory cytokine interleukin-6 (IL-6) and tumor necrosis factor-alpha $(\mathrm{TNF}-\alpha){ }^{2}$ Recent prospective studies have demonstrated that subjects with low grade inflammation have a higher risk of cardiovascular diseases. ${ }^{3-5}$ Several lines of evidence suggest that elevation of CRP may reflect not only local inflammation at atherosclerotic lesions but also systemic 
abnormalities related to insulin resistance, such as increase in fasting insulin, body mass index (BMI), systolic blood pressure and triglyceride $(\mathrm{Tg})$ as well as decrease in high density lipoprotein cholesterol (HDL-C) ${ }^{6-8}$

Studies have shown high level of high sensitive C- reactive protein (hsCRP) in subjects with type 2 diabetes mellitus and dyslipidemia. Data are, however, lacking regarding lipidemic status and hsCRP level in a well-defined group of healthy subjects in Bangladeshi population. In this perspective current study was done to measure hsCRP in the serum of Bangladeshi healthy adults and to explore its association with their lipidemic status.

\section{MATERIALS AND METHODS}

An invitation was made to the volunteer of the study through personal contact to report in the department of Biochemistry and Physiology, Bangladesh Institute of Research \& rehabilitation for Diabetes, Endocrine and Metabolic Disorders (BIRDEM) and requested to refrain from fatty meal for $24 \mathrm{hrs}$ preceding date of appointment. Subjects reported were examined for their wellbeing. Purpose and nature of the study were explained to them. Consented respondents were give appointment for blood sampling. Detailed medical and personal history was recorded on the day of blood sampling in a predesigned case record form.

\section{Anthropometric measurements and blood pressure recording}

Volunteer's height (in meter) and weight $(\mathrm{kg})$, waist and hip $(\mathrm{cm})$ circumference were taken following standard procedure. Cut-off values for BMI (normal $<22.9 \mathrm{Kg} / \mathrm{m} 2$; over weight 23-27.5 and Obese $<27.5$ ) and WHR (male0.90 and female-0.80) were used as per WHO guidelines for Asian population (WHO 2004). Blood pressure (average of two independent measurements) was recorded using barometric Sphygmomanometer.

Five milliliter of venous blood was drawn from each subject by vein puncture at a fasting state. After 20 minutes samples were centrifuged at $3000 \mathrm{rpm}$ for 10 minutes. Separated serum was aliquoted in microcentrifuge tubes, labeled and preserved at $-30 \mathrm{oC}$ for biochemical analyses.

Table 1: Distribution of study subjects on the basis of
different hsCRP level
\begin{tabular}{llcc}
\hline Concentration of & hsCRP(mg/l) & Number & $\%$ \\
\hline hsCRP $<\mathbf{1}(\mathbf{m g} / \mathbf{l})$ & 38 & $(25.5 \%)$ \\
hsCRP $1-3(\mathbf{m g} / \mathbf{l})$ & 64 & $(43.0 \%)$ \\
\hline hsCRP>3 & $(\mathbf{m g} / \mathbf{l})$ & 47 & $(31.5 \%)$
\end{tabular}

When $3 \mathrm{mg} / \mathrm{l}$ is taken as the cut off value of hsCRP, subjects with more hsCRP had significantly high triglyceride value and lower $H D L-c$ value compared to the normal counterparts.

\section{Biochemical methods}

Glucose was measured by (glucose-oxidase) and total cholesterol, triglyceride and HDL cholesterol was measured (by enzymatic colorimetric) method using in the Biochemistry Aut-analyzer 'Hitachi 704' reagents of RANDOX Laboratories Ltd., UK. LDL- cholesterol was calculated using Friedwald formula (Friedewald et al., 1972). The method was not applied when triglyceride level exceeded $400 \mathrm{mg} / \mathrm{dL}$. SGPT by UV spectrophotometric method, Serum creatinine by alkaline picrate method Serum hsCRP by enzyme linked immunosorbant assay (ELISA) method.

\section{Statistical methods}

Data were expressed as mean $\pm \mathrm{SD}$ and number (percent). Statistical analyses were performed using Statistical Package for Social Science (SPSS) for Windows Version 10. Two tailed P value 0.05 was taken as significant level.

\section{Ethical Consideration}

The Helsinki Declaration on medical ethics was respected in the surveys. The protocol was approved by the Ethical Committee of Diabetic Association of Bangladesh.

\section{RESULTS}

The mean age of the participants was 40.4 years. Of the total 149 subjects $92(61.7 \%)$ and $57(38.2 \%)$ were male and female respectively. The mean value of hsCRP was 2.46 $\mathrm{mg} / \mathrm{dl}$. When the study subjects (149) were sub-grouped on the basis of different hsCRP cut off values, $25.5 \%$ had hsCRP $<1 \mathrm{mg} / \mathrm{dl}, 43.0 \%$ between $1-3 \mathrm{mg} / \mathrm{dl}$ and $31.5 \%>3$ $\mathrm{mg} / \mathrm{dl}$ (Table 1).

Results were expressed as mean \pm SD. Unpaired Student's $\mathrm{t}$ test was performed to compare between groups. HDL-c, High density lipoprotein cholesterol; LDL-c, Low density lipoprotein cholesterol; hsCRP, High sensitive C-reactive protein (Table 2).

The study subjects were categorized on the basis of cut-off values with lipids. Of the total subjects $37.8 \%$ subjects had

Table 2: Clinical and biochemical variables of study subjects based on the cut off value of hSCRP $(n=149)$

\begin{tabular}{lclc}
\hline & $\begin{array}{c}\text { Low hsCRP } \\
\mathbf{( 1 0 2 )}\end{array}$ & $\begin{array}{l}\text { High hsCRP } \\
\mathbf{( 4 7 )}\end{array}$ & t/p value \\
\hline Fasting glucose (mmol/l) & $5.1 \pm 0.5$ & $5.2 \pm 0.6$ & $\mathrm{Ns}$ \\
\hline Triglycerides (mg/dl) & $136 \pm 72$ & $164 \pm 81$ & $-2.152 / 0.033$ \\
\hline Total Cholesterol (mg/dl) & $185 \pm 36$ & $183 \pm 35$ & $\mathrm{Ns}$ \\
\hline HDLc (mg/dl) & $38.2 \pm 7.6$ & $34.5 \pm 6.5$ & $2.931 / 0.004$ \\
LDLc (mg/d) & $117 \pm 30$ & $124 \pm 36$ & $\mathrm{Ns}$ \\
\hline
\end{tabular}

Low hsCRP, hsCRP $<3 \mathrm{mg} / \mathrm{l} ; \mathrm{hs} C R P, h s C R P \geq 3 \mathrm{mg} / \mathrm{l}$. 
Table 3: Spearman's correlation analysis for hsCRP with independent variables $(n=149)$

\begin{tabular}{lcc}
\hline \multicolumn{1}{c}{ Variables } & r & P \\
\hline Fasting glucose & 0.030 & 0.716 \\
Triglyceride & 0.171 & 0.037 \\
Total Cholesterol & 0.016 & 0.842 \\
\hline HDL-c & -0.157 & 0.056 \\
\hline LDL-c & 0.150 & 0.067 \\
Smoking & 0.083 & 0.318
\end{tabular}

Results were expressed as Spearman's correlation and statistical significance $p$.

$H D L-c$, High density lipoprotein cholesterol; LDL- c, Low density lipoprotein cholesterol.

Table 4: Binary logistic regression analysis taking hsCRP as dependent and WHR, triglyceride, HDL-c and total cholesterol as independent variables $(n=149)$

\begin{tabular}{lcccccc}
\hline \multicolumn{1}{c}{ Variables } & B & SE & Wald & df & Sig & $\operatorname{Exp(B)}$ \\
\hline Age & -.015 & .025 & 0.380 & 1 & 0.537 & 0.985 \\
Gender & 0.236 & 0.402 & 0.346 & 1 & 0.556 & 1.267 \\
BMI & -0.031 & 0.058 & 0.289 & 1 & 0.591 & 0.969 \\
Triglyceride (mg/dl) & 0.005 & 0.003 & 2.461 & 1 & 0.117 & 1.005 \\
HDL-c (mg/dl) & -0.061 & 0.028 & 4.792 & 1 & 0.029 & 0.969
\end{tabular}

lipid abnormality (TG, TC and LDL-c above and HDL-c below the cut-off value).

Separately:

Hypertriglyceridemia (>150 mg/dl) - 54 (35.5\%)

Hypercholesterolemia (>200 mg/dl) - had 36 (23.7\%)

HDL-c (Male; $<40 \mathrm{mg} / \mathrm{dl}$ and Female; $<50 \mathrm{mg} / \mathrm{dl}$ ) -117 $(77 \%)$

LDL-c (>130 mg/dl) - 51 (34.2\%).
Spearman's correlation analyses were performed for variable fasting glucose, TG, Total Cholesterol, HDL-c and LDL-c. Triglyceride showed positive correlation $(\mathrm{r}=0.171$ $\mathrm{p}=0.037)$ and HDL-c showed negative correlation ( $\mathrm{r}=-$ $0.157 \mathrm{P}=0.056$ ) with hsCRP (Table 3).

Logistic regression was performed taking hsCRP group as dependent variable and age, gender, BMI, Triglyceride and HDL-c as independent variables.

Age $(p=0.537)$, Gender $(p=0.556)$, BMI $(p=0.591)$, Triglyceride $(\mathrm{p}=0.117)$ did not show any significant association with hsCRP. HDL-c showed significant $(B=$ $-0.061, p=0.029)$ negative association with hsCRP. But if HLD-c was omitted from the list of independent variables and smoking was added, triglyceride showed significant positive association $(\mathrm{p}=0.037)$ (Table 4 and 5$)$.

\section{DISCUSSION}

High sensitive C-reactive protein has been a subject of interest among the researchers in recent times. By definition it is the detection of very low level of CRP in the blood using sensitive ELISA or immunoturbidometry method. Usually CRP is present in the blood of healthy person at a very low level.

It is widely regarded that CRP is the biochemical marker of ongoing inflammatory process and long been used to evaluate prognosis by the clinicians. Detection of very low level of CRP 'hsCRP' in the blood has been regarded as the presence of subclinical inflammation. Elevated level of hsCRP has been found to be associated with cardiovascular diseases by a number of cross sectional studies ${ }^{9,10}$ and data of some longitudinal studies have provided substantial evidence for hsCRP to be a predictor of CVDs. ${ }^{11,12}$ It may be mentioned that metabolic disorders, which included obesity, diabetes and hypertension are affecting the mankind irrespective of rich and poor, race and geographical area. ${ }^{13}$ Abnormalities in blood lipids have been implicated in

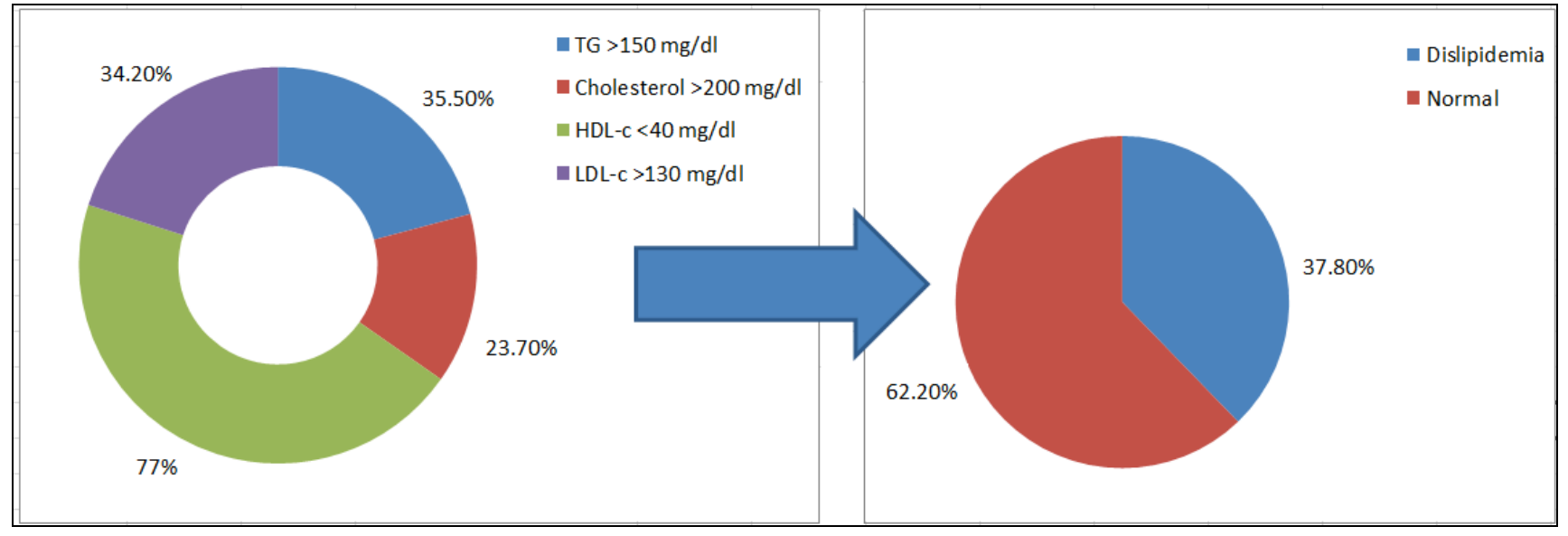

Figure 1: Pie chart showing proportion of subjects with lipid abnormalities 


\begin{tabular}{|c|c|c|c|c|c|c|}
\hline Variables & B & $\mathbf{S E}$ & Wald & df & Sig & Exp(B) \\
\hline Gender & -1.157 & 0.685 & 2.852 & 1 & 0.091 & 0.314 \\
\hline BMI & -0.092 & 0.195 & 0.220 & 1 & 0.639 & 0.91 \\
\hline Triglyceride & 0.010 & 0.005 & 4.361 & 1 & 0.037 & 1.010 \\
\hline Cholesterol & -0.010 & 0.012 & 0.742 & 1 & 0.389 & 0.990 \\
\hline Smoking & 0.525 & 0.810 & 0.421 & 1 & 0.516 & 1.691 \\
\hline
\end{tabular}

Variables entered: Gender, BMI, Triglyceride, Cholesterol, Smoking,

the inflammatory process and pathogenesis of metabolic disorders particularly cardiovascular disorders. Both cross sectional and prospective studies have documented substantial evidence in this regard. ${ }^{14,15}$ However, the nature and extent of inflammation seen to vary depending on ethnic, cultural and environmental background of a particular population. The main focus of the present study was to determine hsCRP in a group of adult healthy subjects of Bangladeshi origin and explore its relationship with lipid levels.

It demonstrates that the mean $\pm \mathrm{SD}$ hsCRP levels of study subjects are $2.46 \pm 1.89$. Although the number of subjects was relatively small, it gives an approximate baseline data for the Bangladeshi population. In previous studies on the same population; mean hsCRP $1.8(\mathrm{mg} / \mathrm{l})$ in healthy controls and $4.0(\mathrm{mg} / \mathrm{l})$ in type2 DM patients were found. Another case control study found hsCRP mean $2.14 \pm 0.13$ in healthy controls of Bangladesh. ${ }^{16}$ Compared to the study involving Rajasthanis in India the mean value in the present study appeared to be high 10 which is probably consistent with those involving Chinese. ${ }^{17}$ It may be mentioned that among the Saudis mean hsCRP level was found to be 2.7, which substantiate the view that race and geographic distribution influence the level.

Taking the conventional upper limit for hsCRP $(<3 \mathrm{mg} / \mathrm{l})$ in consideration $31.5 \%$ (47 out of 149 ) of subjects are found to have high hsCRP. It is further observed that $43 \%$ (64 out of 149) study subjects have hsCRP between $1-3 \mathrm{mg} / \mathrm{l}$ and only $25.5 \%$ have $<1 \mathrm{mg} / \mathrm{l}$ of hsCRP. The findings highlight that even among the healthy subject $41.5 \%$ have hsCRP above the cutoff levels. This may be explained by the presence of ongoing low grade inflammation in these subjects.

Lipid abnormalities are generally found to be associated with cardiovascular disorders which again linked to subclinical inflammation. The present study demonstrates that mean lipid levels are within the normal level [National Cholesterol Education Program, 2001]. However when cutoff values for triglyceride, total cholesterol, LDL-c, HDL-c are considered, $35.5 \%$ are found to have hypertriglyceridemia $(>150 \mathrm{mg} / \mathrm{dl}), 23.7 \%$ hypercholesterolemia $(>200 \mathrm{mg} /$ dl), 77\% low HDL-c (Male; $<40 \mathrm{mg} / \mathrm{dl}$ and Female; $<50$ $\mathrm{mg} / \mathrm{dl}$ ) and $34.2 \%$ have high LDL-c (>130 mg/dl) (fig. 1).
This finding also highlights the fact that among apparently healthy subjects a substantial proportion of subjects have dyslipidemia of individual component. The study also reveals that $37.8 \%$ of the study subjects have one or more lipid abnormalities.

The present study also demonstrates that subjects with hsCRP above the cutoff have significantly high triglyceride value ( $p=0.033$ ) and lower HDL-c value $(\mathrm{p}=0.004)$ compared to the normal counterparts. It is understood that triglyceride is implicated in the pathogenesis of CVDs which is again regarded as the resultant of chronic inflammation. This relationship between hsCRP and triglyceride and HDL-c deserves further analysis. Serum triglyceride was found to have significant positive correlation with hsCRP $(\mathrm{p}=0.03)$.

Serum HDL-c values in healthy Bangladeshi adults appear to be at the lower limit of the normal range as evidenced by other studies in BIRDEM with hsCRP. Serum hsCRP showed a marginal negative association with HDL-c $(\mathrm{p}=0.056)$. It resembles other studies in several populations. ${ }^{10,17}$ The relationship between TG and hsCRP is consistent with other studies. ${ }^{7,10,17}$ Binary logistic regression analysis revealed the relationship when adjusted with gender, BMI and cholesterol level. However in the model when both triglyceride and HDL were present significant negative correlation was observed. This confirms that hsCRP is associated with triglyceride and HDL-c.

Our study was an attempt to extensively analyze the inflammatory marker (hsCRP) with respect to their association with individual lipids and other risk factors to help to identify individuals at higher risk for cardiovascular events despite not meeting criteria based on lipid profile or other traditional risk factors alone. Further large scale multicenter studies with different anthropological parameters are needed to consolidate these preliminary results in a large population.

\section{CONCLUSION}

From the data it may be concluded that i) More than one-third healthy Bangladeshi adults may have chronic subclinical inflammation and thus they may have an additional risk of cardiovascular disorders; and ii) About one-third healthy Bangladeshi adults have one or more type of dyslipidemia; and iii) The subclinical chronic inflammation in healthy Bangladeshi adults has a positive association with their serum triglyceride and negative association with their HDLcholesterol levels.

\section{Acknowledgement}

We acknowledge the important contribution of the volunteers, laboratory staff, Biomedical Research Group of BIRDEM, to make this work possible. Special thanks to the Diabetic Association of Bangladesh (BADAS) for financial 
support. But of all we are grateful to all the subjects who participated in the study.

\section{REFERENCES}

1. 1. Schultz DR, Arnold PI. Properties of four acute phase proteins: C-reactive protein, serum amyloid A protein, alpha 1-acid glycoprotein, and fibrinogen. Semin Arthritis Rheum 1990;20:12947. Crossref

2. Uhlar CM, Whitehead AS. Serum amyloid A, the major vertebrate acute-phase reactant. Eur J Biochem 1999;265:501-23. Crossref

3. Ridker PM, Buring JE, Shih J, Matias M, Hennekens CH. Prospective study of C-reactive protein and the risk of future cardiovascular events among apparently healthy women. Circ 1998;98:731-33. Crossref

4. Kuller LH, Tracy RP, Shaten J, Meilahn EN: Relation of C-reactive protein and coronary heart disease in the MRFIT nested case-control study. Multiple Risk Factor Intervention Trial. Am J Epidemiol 1996;144:537-47. Crossref

5. Sato H, Miida T, Wada $\mathrm{Y}$ et al. Atherosclerosis is accelerated in patients with long-term well-controlled systemic lupus erythematosus (SLE). Clin Chim Acta 2007;385:35-42. Crossref

6. Hanyu O, Miida T, Kosuge K et al. Preheparin lipoprotein lipase mass is a practical marker of insulin resistance in ambulatory type 2 diabetic patients treated with oral hypoglycemic agents. Clin Chim Acta 2007;384:118-23. Crossref

7. Ridker PM, Buring JE, Cook NR, Rifai N. C-reactive protein, the metabolic syndrome and risk of incident cardiovascular events: an 8-year follow-up of 14,719 initially healthy American women. Circulation 2003;107:391-7. Crossref

8. Yudkin JS, Stehouwer CD, Emeis JJ, Coppack SW: C-reactive protein in healthy subjects: associations with obesity, insulin resistance, and endothelial dysfunction: a potential role for cytokines originating from adipose tissue. Arterioscler Thromb Vasc Biol 1999;19:972-8. Crossref

9. Pearson TA, Mensah GA, Alexander RW, Anderson JL, Cannon RO 3rd, Criqui M, et al. Markers of inflammation and cardiovascular disease: Application to clinical and public health practice: a statement for healthcare professionals from the Centers for Disease Control and Prevention and the American Heart Association. Circulation 2003;107:499-511. Crossref

10. Sudha Thakur, Shallu Gupta, Hundal Parchwani, Vidhi Shah and Vandana Yadav. Hs-CRP - A Potential Marker for Coronary Heart Disease. Indian Journal of Fundamental and Applied Life Sciences. Crossref

11. Ridker PM. C-reactive protein and the prediction of cardiovascular events among those at intermediate risk: moving an inflammatory hypothesis toward consensus. J Am Coll Cardiol 2007;49:2129-38. Crossref

12. Rifai N, Ridker PM. Inflammatory marker and coronary heart disease. Curr Opin Lipidol 2002;13:383-9. Crossref

13. Nishida M, Moriyama T, Yoshiro Sugita Y, Keiko Yamauchi-Takihara $\mathrm{K}$ : Interleukin-10 associates with adiponectin predominantly in subjects with metabolic syndrome. Circ J2007;71:1234-8. Crossref

14. Maryam Tohidi, Masumeh Hatami, Farzad Hadaegh1, Maryam Safarkhani, Hadi Harati, Fereidoun Azizi. Lipid measures for prediction of incident cardiovascular disease in diabetic and nondiabetic adults: results of the 8.6 years follow-up of a population based cohort study. Lipids in Health and Disease 2010;9:1-9. Crossref

15. Pradhan AD; Manson, JE; Rifai, N; Buring, JE; Ridker, PM (2001). "C-reactive protein, interleukin 6, and risk of developing type 2 diabetes mellitus". JAMA 2001;286:327-34. Crossref

16. Awan ZA, Naveed AK, Malik MM and Naveed A. Evaluation of C-Reactive Protein and Interleukin -6 as Markers of Coronary Heart Disease. ANNALS 2008;14:50-6. Crossref

17. Zhang JQ, Thomas TR, Ball SD. Effect of exercise timing on postprandial lipemia and HDL cholesterol subfractions. J Appl Physiol 1998;85:1516-29. PMid:9760349 